\title{
Augmenting Television With Augmented Reality
}

DOI:

10.1145/3317697.3325129

\section{Document Version}

Accepted author manuscript

Link to publication record in Manchester Research Explorer

\section{Citation for published version (APA):}

Saeghe, P., Clinch, S., Weir, B., Glancy, M., Vinayagamoorthy, V., Pattinson, O., Golds, P., Pettifer, S., \& Stevens, R. (2019). Augmenting Television With Augmented Reality. In ACM International Conference on Interactive Experiences for Television and Online Video https://doi.org/10.1145/3317697.3325129

\section{Published in:}

ACM International Conference on Interactive Experiences for Television and Online Video

\section{Citing this paper}

Please note that where the full-text provided on Manchester Research Explorer is the Author Accepted Manuscript or Proof version this may differ from the final Published version. If citing, it is advised that you check and use the publisher's definitive version.

\section{General rights}

Copyright and moral rights for the publications made accessible in the Research Explorer are retained by the authors and/or other copyright owners and it is a condition of accessing publications that users recognise and abide by the legal requirements associated with these rights.

\section{Takedown policy}

If you believe that this document breaches copyright please refer to the University of Manchester's Takedown Procedures [http://man.ac.uk/04Y6Bo] or contact uml.scholarlycommunications@manchester.ac.uk providing relevant details, so we can investigate your claim.

\section{OPEN ACCESS}




\title{
Augmenting Television With Augmented Reality
}

\author{
Pejman Saeghe ${ }^{1,2}$ \\ Sarah Clinch \\ Bruce Weir ${ }^{2}$ \\ Maxine Glancy ${ }^{2}$ \\ ${ }^{1}$ University of Manchester \\ Manchester, UK \\ forename.surname@manchester.ac.uk
}

\author{
Vinoba Vinayagamoorthy ${ }^{2}$ \\ Ollie Pattinson ${ }^{2}$ \\ Stephen Pettifer ${ }^{1}$ \\ Robert Stevens ${ }^{1}$ \\ ${ }^{2} \mathrm{BBC} R \& D$ \\ Salford, UK \\ forename.surname@bbc.co.uk
}

\begin{abstract}
This paper explores the effects of adding augmented reality (AR) artefacts to an existing TV programme. A prototype was implemented augmenting a popular nature documentary. Synchronised content was delivered over a Microsoft HoloLens and a TV. Our preliminary findings suggest that the addition of AR to an existing TV programme can result in creation of engaging experiences. However, presenting content outside the traditional TV window challenges traditional storytelling conventions and viewer expectations. Further research is required to understand the risks and opportunities presented when adding AR artefacts to TV.
\end{abstract}

\section{KEYWORDS}

Television; Augmented Reality; User Study; User Experience; User Engagement; Human-Media Interaction.

\section{TVX2019, June 2019, Manchester, UK}

(c) 2019 Association for Computing Machinery.

This is the author's version of the work. It is posted here for your personal use. Not for redistribution. The definitive Version of Record was published in Proceedings of ACM TVX (TVX2019), https://doi.org/10.475/123_4. 


\section{Sidebar 1: Questionnaires}

UES-SF The User Engagement Scale Short Form consists of twelve items measuring four constructs: aesthetic appeal, focused attention, perceived usability, and reward [10].

UEQ The user experience questionnaire consists of twenty six items measuring six constructs: attractiveness, perspicuity, efficiency, dependability, stimulation, and novelty [7].

\section{Sidebar 2: Interview Protocol}

1. The overall impression

2. Thoughts on individual holograms regarding each artefact's positioning, visual quality, animation, and potential user interactions

3. Suitable TV genres for this use-case

4. The context in which the participants thought it would make sense to watch such experiences in terms of location, time of day, social context, etc.

5. Thoughts regarding interactive vs passive nature of the artefacts and the experience as a whole

6. Time of appearance of the holograms in relation to the content on TV

7. Other devices for presenting the AR content such as HHD and projection

\section{INTRODUCTION}

The success of augmented reality (AR) in the military, medical, and industrial settings can be attributed to the affordances it provides in making task-related operations more efficient. For instance, in repairing complex machinery AR may be used to reduce task completion time [4] and in assembly lines it may be used to reduce error rate and mental effort [12]. So far, for the general public, AR has achieved relatively small success, compared to that in industro-milarity contexts, primarily in gaming and advertising. However, similar to the internet-which was first developed, tested and used in the military for a number of years before finding its way eventually to (almost) every household and work environment-AR may find widespread use in daily life in the near future.

The British Broadcasting Corporation Research \& Development department (BBC R\&D) and the University of Manchester School of Computer Science have been working together to investigate the feasibility and various design considerations of applying AR in TV. One use-case is to add AR content to an existing TV programme.

A few broadcasters, media companies, and researchers have looked at the application of AR in TV. Kawakita and Nakagawa designed a system called Augmented TV where an animated character appeared to emerge from a TV screen when viewed using a hand-held device (HHD) [6]. However, their work is primarily concerned with technical implementation details. Lack of a user evaluations is another limitation of their work.

Revelle et al. created Electric Agents; an AR game layered on top of an existing educational TV programme, aimed at children between 6 and 10 years of age. The experience took the viewers back and forth between a passive TV-watching mode and an active game-playing mode. The passive mode would be interrupted by a TV character zapping words out of the protagonist's mouth. This marked the beginning of the game-playing mode where children in a pair would use HHDs looking around the room in order to find those words in both their visual and written representations. The children then used the HHDs to "throw" the matched pair back into the TV [11]. This study shows a potential for creating engaging experiences by mixing the passive TV viewing mode with an active AR-enabled game-playing mode. However, this use-case ignores the potential for creating passive immersive experiences using AR.

In [2] Fradet et al. presented a system called MR TV Mosaik where a virtual mosaic is displayed next to the TV enabling: 1) visualisation of other available programmes, 2) switching to a different program via drag and drop from the virtual mosaic to the TV screen, 3) access to programme guides and other textual information about the programme, 4) virtual subtitles, and 5) 3D objects. Their system is focused on providing control and extra information via AR. This misses the opportunity to use the affordances of AR in a meaningful way. For instance, using the interaction provided by AR to 


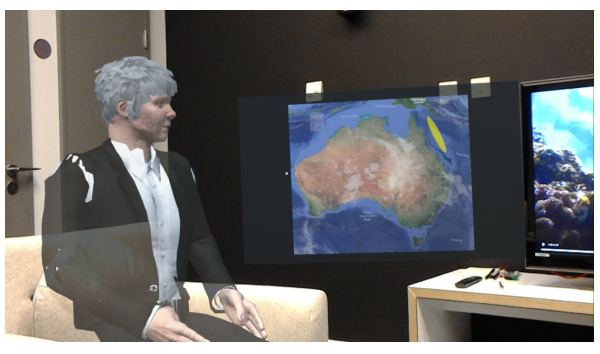

Figure 2: Virtual person sitting on an armchair next to the viewer.

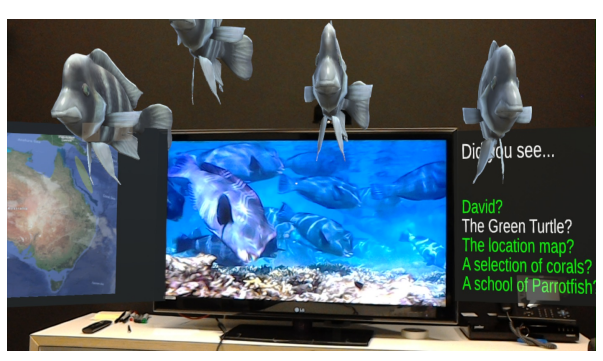

Figure 3: Virtual fish as they swim towards the viewer.

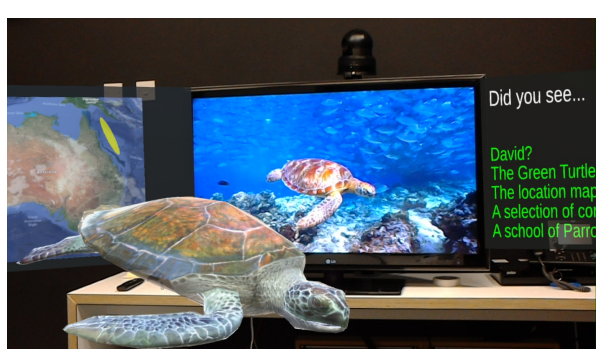

Figure 4: Virtual sea turtle crossing the focal point of the viewer with the two virtual side screens in the background. drive the story instead of changing the channel. Furthermore, these operations can be done using non-AR technology. The lack of a user-based evaluation is another limitation of their work.

In this paper we focus on creating an AR TV experience and consider the following research question:

RQ How does augmenting an existing TV programme, using passive AR artefatcs, affect the viewer's experience and engagement?

In order to answer this research question we created a prototype and evaluated it in a user study using two questionnaires - the User Engagement Scale Short Form (UES-SF) and the User Experience Questionnaire (UEQ) [Sidebar 1] - and a semi-structured interview [Sidebar 2]. Overall the scores were high for all constructs of the UES-SF [Figure 5]. On the UEQ efficiency and dependability scores were close to neutral while the scores for the remaining constructs were higher [Figure 6].

\section{PROTOTYPE}

Our prototype delivered content to the viewer via two platforms: the main content was delivered on the TV screen, while the AR content was viewable using the HMD (a Microsoft HoloLens). Playback of TV and holographic content was synchronised using HbbTV 2.0's sync functionality [9]. The orchestration of the AR content relative to the TV content can be seen in Figure 1.

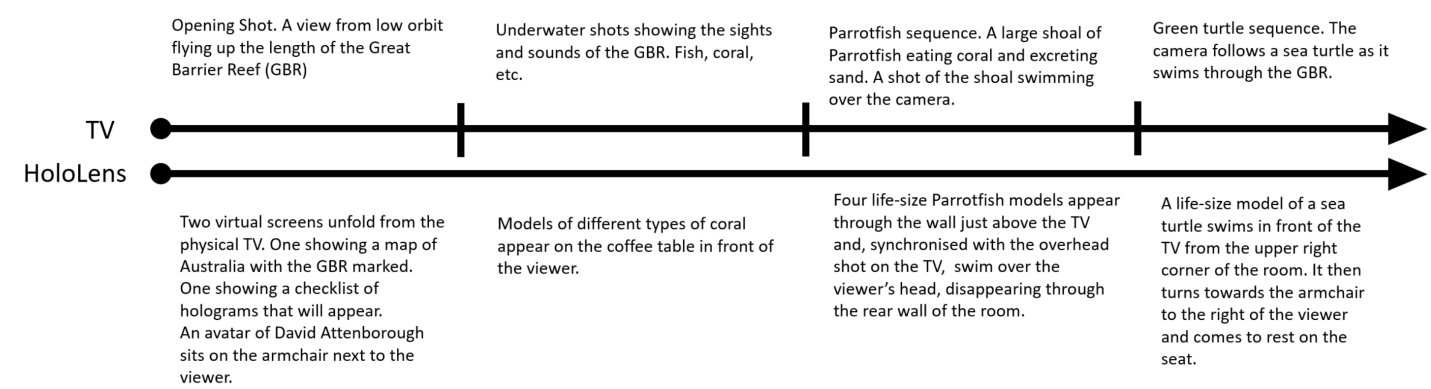

Figure 1: AR TV timeline.

For the main content, we chose a five-minute video clip from the BBC's Blue Planet programme; a popular documentary exploring the world's oceans. The story in the clip took place around the Great Barrier Reef (GBR) and followed the activities of a female sea turtle.

For the AR content we chose a variety of artefacts in order to evoke conversations in the interviews about AR TV possibilities. The AR artefacts used were: 


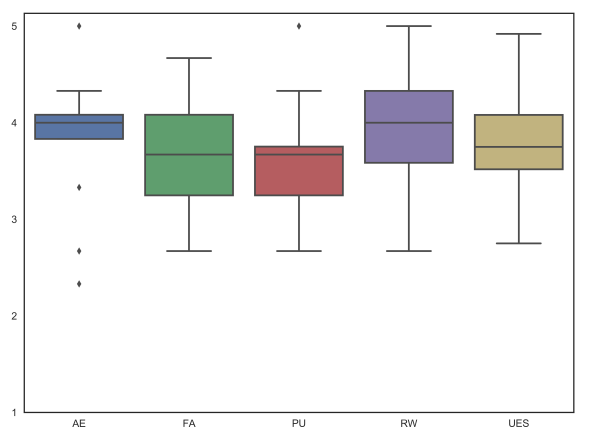

Figure 5: Aesthetic Appeal (AE), Focused Attention (FA), Perceived Usability (PU), Reward (RW) and overall engagement (UES) score distributions for the UES-SF.

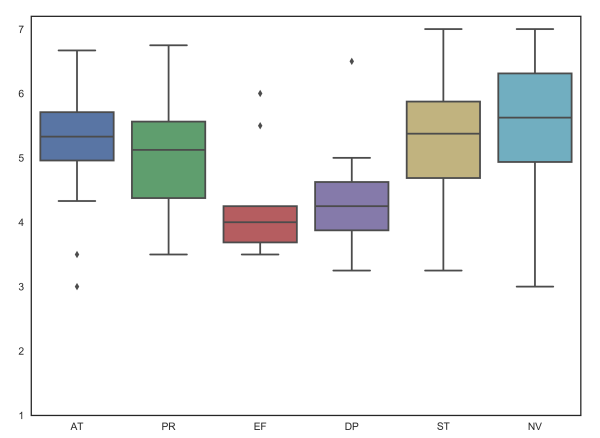

Figure 6: Attractiveness (AT), Perspicuity (PR), Efficiency (EF), Dependability (DP), Stimulation (ST) and Novelty (NV) score distributions for the UEQ.
1) Two virtual screens, either side of the TV, that delivered contextual and feedback information to the viewer. Context about the location of the GBR was conveyed by showing a map of Australia with the GBR area being highlighted. Feedback consisted of the list of holograms due to appear during the experience [Figures 3 and 4].

2) A life-size virtual human male with idle animation, sitting on a real armchair in the viewer's environment, who appears to look at the TV screen and makes eye contact as the viewer looks at him [Figure 2].

3) A set of holograms related to the main content. Corals appeared on a coffee table in front of the viewer. A school of parrot fish appeared above the TV and swam towards the viewer, eventually passing over their head [Figure 3]. A sea turtle appeared near the TV, swam a curved path towards the viewer settled next to them on the sofa [Figure 4].

\section{USER STUDY}

We conducted a user study with a sample of 12 BBC R\&D members of staff. We followed the BBC's internal ethics procedures, with all participants providing informed consent. The study was conducted in a user-testing lab designed to look and feel like a living room with sofas, armchairs, a coffee table and a smart TV. Each participant attended a session that started with a brief introduction to the research. They were then given a few minutes to familiarise themselves with the HoloLens device and to adjust it for comfort. After watching the programme, they were asked to remove the HoloLens and complete the two questionnaires [Sidebar 1].

After completing the questionnaires participants engaged in a semi-structured interview conducted by a member of the research team. The main points in the interview were captured in a protocol as presented in Sidebar 2.

The total engagement required from each participant was approximately one hour.

\section{RESULTS}

UES-SF results indicated that, in general, all measured constructs scored broadly the same, with a moderately high value of 3.5 - 4 [Figure 5]. Most people scored very close to the median on aesthetic appeal with a few clear outliers. Spread for focused attention and reward was much bigger.

UEQ results indicate a big spread of scores for stimulation, novelty and to a slightly lesser extent for perspicuity. These results also indicate agreement around 4 (the neutral score on a 7 point Likert scale) for dependability and a tighter agreement around the same value for efficiency. All the other constructs score slightly higher [Figure 6].

For both questionnaires a larger sample size would yield more reliable readings. 
Analysis of the interviews indicate that overall participants found the experience novel and engaging. The favourite hologram was the sea turtle. Some people found the virtual person unsettling. Most frequent responses about suitable genre were sport and news. Many people felt the scenario would work best when they are on their own, while some thought it would be interesting to share the experience with others. Some people found the passive nature of the experience suitable and were not expecting to be able to interact with the AR content; "it did not even enter my head to try and do something, and really, I don't know if I would have wanted to". The fixed orchestration of the AR and TV content divided opinion; some liked it while others wanted to be able to pause the TV content to view and explore AR artefacts at their own pace. Most people were happy to have their hands free, therefore preferring HMD and projection based AR over HHD. Fear of missing out was raised a few times. For some people this was about missing AR content, while for others this was about missing TV content. Two people reported not seeing the AR sea turtle and the AR fish.

\section{DISCUSSION}

Our findings suggest that augmenting an existing TV programme with AR artefacts can result in increased engagement [Figure 5]. This finding is corroborated by the results of UEQ [Figure 6] which suggest our prototype was perceived as novel, stimulating, aesthetically appealing and perspicuous. The neutral scores for efficiency and dependability could be interpreted in two ways:

1) These constructs are not relevant to a passive AR TV hybrid.

2) Participants found the prototype not dependable or efficient.

Another finding suggests that people may miss AR content that is presented outside the TV window. In our study two participants missed the virtual sea turtle and the virtual fish. This phenomenon could be interpreted in multiple ways:

1) Inattentioanl Blindness (IB): The stimuli presented outside the primary zone of attention (i.e. the TV screen) may fall under IB [8].

2) Novice participants: Most of the participant had very little, if any, prior exposure to AR and none had watched an AR TV experience before. There is some evidence suggesting that lack of experience, as oppose to lack of attention, may cause missing of visual content [1].

3) Missing conventions for AR TV: Each of the currently mature media (as well as each genre within each media) has gone through an initial phase of trial and error in order to establish conventions. For instance, fans of the horror genre know what to expect when they go to the cinema to watch a horror film. Conversely, since no conventions yet exist for AR TV, viewers could simply be missing content because they don't know what to expect. 


\section{FUTURE WORK}

We used various types of holograms to augment a natural documentary programme. Future research is required to understand the effects of each type of AR artefact on viewer engagement.

Experimentation with different genres of TV programmes is required to understand how augmentation affects viewer engagement regarding other genres.

Creating a story from scratch for AR TV would yield useful insights and provide the opportunity to test how the affordances of AR could be mixed with TV to create engaging experiences.

Objective measures such as eye-tracking and user interaction data can be used to provide a different and perhaps more granular insight into viewer engagement.

Other subjective questionnaires could be used for instance to measure immersion with [5] and cognitive load with [3].

\section{CONCLUSION}

AR is likely to become ubiquitous in the near future. The application of AR to enhance conventional TV viewing can result in engaging, stimulating and novel experiences. However, there are risks and issues involved with delivering content in this hybrid format that needs to be understood by further research and exploration.

\section{ACKNOWLEDGMENTS}

Pejman Saeghe is a research student funded through an EPSRC iCASE studentship with the BBC. The authors also acknowledge partial funding from the UK EPSRC under grant number EP/N028228/1 (PACTMAN).

\section{REFERENCES}

[1] Jochen Braun. 1998. Vision and attention: the role of training. Nature 393, 6684 (1998), 424.

[2] Matthieu Fradet, Caroline Baillard, Anthony Laurent, Tao Luo, Philippe Robert, Vincent Alleaume, Pierrick Jouet, and Fabien Servant. 2017. [POSTER] MR TV Mozaik: A New Mixed Reality Interactive TV Experience. In Adjunct Proc. of ISMAR 2017. IEEE, 155-159.

[3] Sandra G Hart and Lowell E Staveland. 1988. Development of NASA-TLX (Task Load Index): Results of empirical and theoretical research. In Advances in psychology. Vol. 52. Elsevier, 139-183.

[4] Steven Henderson and Steven Feiner. 2011. Exploring the benefits of augmented reality documentation for maintenance and repair. IEEE transactions on visualization and computer graphics 17, 10 (2011), 1355-1368.

[5] Charlene Jennett, Anna L Cox, Paul Cairns, Samira Dhoparee, Andrew Epps, Tim Tijs, and Alison Walton. 2008. Measuring and defining the experience of immersion in games. International journal of human-computer studies 66, 9 (2008), 641-661.

[6] Hiroyuki Kawakita and Toshio Nakagawa. 2014. Augmented TV: An augmented reality system for TV programs beyond the TV screen. In Proc. of. ICMCS 2014. IEEE, 955-960.

[7] Bettina Laugwitz, Theo Held, and Martin Schrepp. 2008. Construction and evaluation of a user experience questionnaire. In Symposium of the Austrian HCl and Usability Engineering Group. Springer, 63-76. 
[8] Arien Mack, Irvin Rock, et al. 1998. Inattentional blindness. Vol. 33. MIT press Cambridge, MA.

[9] Klaus Merkel. 2011. Hybrid broadcast broadband TV, the new way to a comprehensive TV experience. In Electronic Media Technology (CEMT), 2011 14th ITG Conference on. IEEE, 1-4.

[10] Heather L O'Brien, Paul Cairns, and Mark Hall. 2018. A practical approach to measuring user engagement with the refined user engagement scale (UES) and new UES short form. IJHCS 112 (2018), 28-39.

[11] Glenda Revelle, Emily Reardon, Kristin Cook, Lori Takeuchi, Rafael Ballagas, Koichi Mori, Hiroshi Horii, Hayes Raffle, Maria Sandberg, and Mirjana Spasojevic. 2014. Electric agents: Combining collaborative mobile augmented reality and web-based video to reinvent interactive television. Computers in Entertainment (CIE) 12, 3 (2014), 1.

[12] Arthur Tang, Charles Owen, Frank Biocca, and Weimin Mou. 2003. Comparative effectiveness of augmented reality in object assembly. In Proceedings of the SIGCHI conference on Human factors in computing systems. ACM, 73-80. 